\title{
Emergência de Relações Ordinais em Crianças ${ }^{1}$
}

\author{
Grauben José Alves de Assis \\ Livia Cristinne Arrelias Costa
}

Universidade Federal do Pará

\begin{abstract}
RESUMO
Nas relações entre estímulos em seqüências ensinadas independentemente uma da outra, deve-se considerar as propriedades definidoras da ordinalidade: irreflexividade, assimetria, transitividade e conectividade. O Estudo I replicou a emergência de classes ordinais com crianças normais, a partir de treino por encadeamento e o Estudo II ampliou esses resultados sob controle condicional. Um microcomputador forneceu suporte ao estudo. No Estudo I foi programado um procedimento com quatro conjuntos de estímulos não usuais e no Estudo II dois conjuntos de estímulos. Após ordenar um conjunto de estímulos, duas cores foram introduzidas e o responder deveria ocorrer em função da presença das cores "verde" ou "vermelho". A tarefa do participante era tocar em uma figura e depois, na outra. Respostas corretas produziam uma animação gráfica na tela. Atingido o critério de acerto eram aplicados testes para avaliar a ordinalidade, transitividade e conectividade avaliando a emergência de classes ordinais ou de equivalência. Todos participantes responderam no treino. Nos testes de substitutabilidade do Estudo I todos responderam prontamente. No Estudo II, dois participantes responderam prontamente e um parcialmente. Esses resultados indicam que o procedimento utilizado foi efetivo e ampliou os obtidos em estudos anteriores. O presente estudo pode ser visto como importante no sentido de contribuir para análise de comportamentos conceituais numéricos.
\end{abstract}

Palavras-chave: encadeamento; classes ordinais; crianças.

\section{ABSTRACT \\ The Emergence of Ordinal Relations in Children}

In the study of the relationship between stimuli, across sequences trained independently of each other, one should consider the defining properties of common factors such as inflexibility, asymmetry, transivity, and connectedness. Study \#I verified the presence of emergent ordinal classes in normal children by chaining teaching. Study \#2 extended this research to conditional control, utilizing a microcomputer. In the first study, a chaining conditioning procedure was employed with four sets of uncommon stimuli. In study II, two sets of stimuli were used, after ordering a figures set two colors were introduced with responses occurring in the presence of two colors: "green" or "red". Figures appeared side by side on the screen, and the participant touched each in turn. If the figures were correctly ordered, an animated graphic appeared on the screen. After reaching criteria, ordinarily was tested, transivity and connectedness evaluating the emergence of ordinal classes or equivalency. Every participant responded consistently during the training phase. In study I, all participants responded readily to tests of substituitability. In study II, two participants responded readily and one partially. These results suggest that the procedure was effective and replicated findings obtained in a previous study with handicapped students. This investigation made an important contribution to research dealing with the analysis of numerical concepts.

Keywords: chaining; ordinal classes; children.

A expansão da unidade básica da Análise Experimental do Comportamento (AEC) foi documentada a partir de estudos conduzidos por Sidman (cf. 1986; 1994) e Sidman e Tailby (1982) que ampliou a noção de contingência de reforçamento de três para quatro e cinco termos e os processos comportamentais envolvidos nestas relações de dependência.

Em uma discriminação condicional, a função exercida pelos estímulos discriminativos muda de acordo com o contexto no qual aparecem. Em outras palavras, a função de um estímulo discriminativo é alterada conforme a presença ou ausência de outros estímulos denominados estímulos condicionais. Assim, uma discriminação condicional é demonstrada quando um estímulo discriminativo de segunda ordem (estímulo condicional) exerce controle sobre os estímulos discriminativos primários estes, por sua vez, controlam a resposta através da presença do reforço. Um procedimento padrão de discriminação condicional ampla- 
mente usado tem sido o de emparelhamento de acordo com o modelo (matching to sample).

Sidman (1992) apresentou um exemplo muito didático. Um estímulo modelo poderia ser qualquer um de três numerais: 1, 2 ou 3 e os estímulos de comparação, as palavras correspondentes aos numerais escritos na língua inglesa - one, two ou three. Um aluno seria ensinado a relacionar o numeral (modelo) à palavra escrita na língua inglesa correspondente, com reforçamento social. Na segunda condição, os estímulos modelos seriam palavras inglesas correspondentes aos numerais 1, 2 e 3 e os estímulos de comparação, palavras escritas na língua portuguesa correspondentes aos mesmos numerais. Agora seria ensinada ao aluno a relação entre a palavra escrita em inglês com a palavra escrita em português correspondente. Finalmente, uma outra tarefa seria fornecida ao participante: relacionar as palavras portuguesas como estímulos modelos com os numerais 1, 2 e 3 como estímulos de comparação. Após algumas tentativas, o participante seria capaz de estabelecer essas novas relações sem qualquer ensino prévio, sem reforçamento diferencial, ou seja, novos desempenhos emergiriam sem treino explícito.

Em uma situação de matching se exige uma resposta ao modelo e em seguida pelo menos dois estímulos de comparação são apresentados. Tradicionalmente essa resposta (tocar ou apontar) é a mesma para ambos os estímulos sendo que, quando emitida para o estímulo modelo é denominada resposta de observação; e quando apresentada a um dos estímulos de comparação, é denominada resposta de escolha, sendo esta, seguida de reforçamento. A função da resposta de observação, em geral, é garantir a atenção do participante ao estímulo modelo. Ou seja, a relação estímulo-estímulo (S-S) controlaria diretamente a resposta de escolha. Em termos de relações condicionais, ao ensinar a um participante a relação $\mathrm{AB}$ e a relação $\mathrm{BC}$, temos como resultado a emergência das relações $\mathrm{AC}$ e CA.

De acordo com Sidman e Tailby (1982), a formação de classes de equivalência implica em que um organismo aprenda mais do que foi diretamente ensinado: o ensino direto de um conjunto de relações deve produzir desempenhos adicionais não diretamente ensinados.

Uma análise semelhante foi proposta por Green, Stromer e Mackay (1993) para investigar a formação de classes ordinais, como uma extensão do paradigma de equivalência (cf. Sidman, 1994), com ênfase na análise de relações ordinais estabelecidas entre estímulos em uma seqüência ou entre seqüências diferentes.

\section{As relações entre estímulos em classes ordinais}

Green e cols. (1993) descrevem o estudo da formação de classes ordinais como um tipo de análise de desempenhos produtivos com duas características principais: ser uma proposta análoga conceitual e metodologicamente ao paradigma de equivalência de estímulos proposto por Sidman $(1986 ; 1994)$ e ter implicações tanto teóricas como práticas para estudos relacionados com o comportamento verbal (sintaxe e comportamentos conceituais numéricos).

Ordenar envolve sempre uma relação temporal e espacial dos eventos ambientais. Como um organismo pode responder ordinalmente a eventos fisicamente diferentes que se sucedem no tempo e que nunca foram relacionados anteriormente e, o mais relevante: como novas relações ordinais podem emergir sem qualquer treino adicional? Relações ordinais implicam na comparação entre duas situações, nas quais um elemento precede ou sucede outro. Ou seja, organizar objetos em uma sequiência significa dispor esses objetos com base em uma determinada ordem. Green e cols. (1993) propuseram testes comportamentais que avaliam se essas relações apresentam as propriedades definidoras de relações ordinais: irreflexividade, assimetria, transitividade e conectividade, análogas às da matemática (cf. Stevens, 1951).

\section{As propriedades de uma relação ordinal}

A definição matemática de uma relação ordinal foi tomada como guia para uma análise descritiva e organizada de relações estímulo-estímulo produzidas por um treino seqüencial. Para isto, Green e cols. (1993) propuseram códigos alfanuméricos para representar estímulos e o símbolo $\rightarrow$ para indicar uma relação de ordem (algo como "vem antes" com o sinal). Por exemplo, $\mathrm{A} 1 \rightarrow \mathrm{A} 2 \rightarrow \mathrm{A} 3 \rightarrow \mathrm{A} 4 \rightarrow \mathrm{A} 5$ representam cinco estímulos diferentes aos quais se responde numa dada ordem (primeiro, segundo, terceiro, quarto e quinto).

De acordo com a matemática, irreflexividade pressupõe que uma relação ordinal é explicitamente não reflexiva. Não é verdade, por exemplo, dizer que $\mathrm{A} 1 \rightarrow \mathrm{A} 1$. A propriedade da assimetria pressupõe que uma relação ordinal deve ser unidirecional; neste caso, se $A 2 \rightarrow A 3$, então a relação $A 3 \rightarrow A 2$ não pode ser considerada correta. Uma relação ordinal é considerada transitiva se, por exemplo, A2 $\rightarrow$ A 3 e A3 $\rightarrow$ A4, então A2 $\rightarrow$ A4. Note-se que apenas pares de estímulos que não são adjacentes (como por exemplo, A2 $\rightarrow$ A4; A3 $\rightarrow$ A4) dentro de séries treinadas podem ser a base para a inferência desta propriedade. E, finalmente, a propriedade de conectividade é demonstrada em situações em que, se $\mathrm{A} 1 \rightarrow \mathrm{A} 2 \rightarrow \mathrm{A} 3$, então $\mathrm{A} 1 \rightarrow \mathrm{A} 2$, $\mathrm{A} 1 \rightarrow \mathrm{A} 3$ e A2 $\rightarrow$ A3. Ela pressupõe que uma relação é verdadeira se é correspondente entre todos os pares de 
estímulos dentro de um dado conjunto. Como se vê, esta propriedade somente é inferida se todos os pares são possíveis, sejam eles adjacentes ou não adjacentes. Relações deste tipo são necessárias, mas sua identificação não é suficiente para a produção de classes ordinais.

\section{A formação de classes ordinais}

Alguns estudos têm investigado se o ensino de relações ordinais com dois ou mais conjuntos de estímulos estabelecem classes de estímulos que ocupam a mesma posição ordinal (primeiro, segundo, terceiro) em diferentes conjuntos. Seu desenvolvimento é inferido a partir dos desempenhos em testes que avaliam se estímulos de uma mesma posição ordinal em diferentes conjuntos são substituíveis (intercambiáveis) uns pelos outros em classes ordinais. Se positivo, então poderíamos afirmar que há transferência de funções, ou seja, assim como classes de equivalência são também funcionais, classes de sequiência também seriam classes funcionais?

Em um estudo realizado por Stromer e Mackay (1993), crianças e adultos normais foram ensinados a tocar cinco estímulos fisicamente diferentes em uma ordem designada pelo experimentador, independentemente da sua localização na tela do computador. $\mathrm{O}$ treino envolveu quatro classes ordinais com pares de dois estímulos sobrepostos (overlapping two stimuli sequences). A produção de novas classes de dois e cinco estímulos emergiu deste treino. Os novos estímulos funcionaram como membros de classes ordinais existentes e a variação do procedimento de treino de duas classes adjacentes pode fornecer um caminho para isolar e avaliar propriedades transitivas potenciais de relações ordinais entre estímulos.

No estudo conduzido por Holcomb, Stromer e Mackay (1997), os autores usaram o mesmo procedimento de ensino com sobreposição de estímulos. O objetivo do estudo foi verificar se crianças ensinadas a ordenar pares de estímulos visuais também responderiam a novas seqüências sem qualquer treino adicional. Neste estudo, crianças normais de educação infantil foram capazes de responder ordinalmente a três ou mais estímulos, após serem expostas ao treino com dois estímulos sobrepostos. Entretanto, foram incapazes de responder a seqüências longas (Experimento 1). No Experimento 2, os autores complementaram o procedimento anterior com um procedimento de fading (introdução gradativa dos estímulos com diferentes intensidades), buscando reduzir a possibilidade de erros. Seis outros participantes responderam corretamente a novas sequiências com três estímulos. Um terceiro experimento foi conduzido para avaliar se os participantes continuariam respondendo, sem que testes com pares de estímulos adjacentes fossem usados progressivamente, como nos dois estudos anteriores. Quatro crianças normais foram submetidas ao mesmo treino anterior com dois estímulos sobrepostos e fading. Três participantes apresentaram relações de transitividade e de conectividade com até seis estímulos. Segundo Holcomb e cols. (1997) os resultados mostraram que relações de transitividade (responder ordinalmente a pares de estímulos não-adjacentes) foram obtidas, mesmo na ausência de qualquer mediação verbal. Os autores chamaram atenção para a ordem de treino em que os conjuntos foram ensinados.

Em um estudo conduzido numa escola pública especializada para deficientes mentais, Assis e Sampaio (2003) analisaram dois procedimentos de ensino que foram usados para a formação de classes ordinais: encadeamento e sobreposição de estímulos com cinco estímulos visuais. A partir destes procedimentos, os autores puderam inferir o controle de relações de ordem na produção de novas classes de estímulos. Os resultados mostraram que todos os participantes obtiveram $100 \%$ de acertos nos treinos e em todos os testes. O treino por encadeamento foi mais eficiente nos dois grupos experimentais, independentemente da ordem de apresentação dos treinos. A permanência dos estímulos na tela ao longo de todas as tentativas durante o procedimento de encadeamento parece ter contribuído para esses resultados.

Sampaio e Assis (no prelo) realizaram um outro estudo com pessoas portadoras de deficiência mental, procurando investigar se após a emergência de classes ordinais, os estímulos envolvidos seriam funcionalmente equivalentes. Green e cols. (1993) apontaram teoricamente essa possibilidade, mas sem qualquer evidência empírica. Os autores utilizaram o procedimento de treino por encadeamento, avaliando os efeitos de uma história de treino com estímulos usuais e não usuais. Este estudo mostrou a eficiência do procedimento de encadeamento na formação de classes ordinais em portadores de deficiência mental, apresentando dados consistentemente regulares e, pela primeira vez, demonstrou empiricamente que os membros das classes ordinais eram também funcionalmente equivalentes. Segundo Sampaio e Assis (no prelo) "o mesmo processo comportamental envolvido na equivalência de estímulos pode estar também envolvido no desempenho ordinal, e este processo poderia fornecer a base para o desenvolvimento da sintaxe e da ordenação de palavras" (p. 56). Isso poderia proporcionar as bases para o desenvolvimento de uma tecnologia para ensinar desempenhos acadêmicos que envolvem leitura, escrita e habilidades pré-aritméticas, além do ensino de comportamentos adaptativos (por 
exemplo, comportamentos de higiene e de limpeza) para pessoas portadoras de necessidades especiais.

\section{Estudos sobre Controle Condicional em seqüência de estímulos}

Um responder ordinal ainda pode estar sob controle condicional. Por exemplo, na vida cotidiana poderíamos observar, num boletim escolar de um estudante, o seguinte: Carlos tem 10 anos e está na $2^{\mathrm{a}}$ série, Antônio tem 8 anos e está na $1^{\mathrm{a}}$ série e Francisco, de 7 anos, está na $3^{a}$ série do ensino fundamental. Ou seja, poderíamos organizar os nomes dos alunos dependendo da ordem de nascimento ou do nível de escolaridade. Assim teríamos: Francisco, Antônio e Carlos, se o critério de ordenar os nomes fosse pela idade do mais jovem ao mais velho, ou Antônio, Carlos e Francisco, se o critério fosse o nível de escolaridade da primeira para a última série.

Para demonstrar empiricamente esse fenômeno, Lazar e Kotlarchyk (1986) realizaram um estudo cujo objetivo foi determinar se um procedimento de matching poderia produzir seqüências de comportamento não ensinadas diretamente. Nesse estudo, quatro crianças normais que tinham entre 5 e 6 anos completos foram ensinadas a responder em tarefas de matching estabelecendo duas classes de equivalência. Um membro de cada classe já ensinada adquiria propriedades ordinais de "primeiro" e "segundo". Posteriormente, os membros remanescentes das duas classes de equivalência seriam colocados em uma ordem e, sem treino, as crianças conseguiram responder na ordem apropriada. Antes de iniciar o treino com matching as crianças eram ensinadas a selecionar letras gregas relacionadas a dois conjuntos de estímulos. As letras gregas foram escolhidas por se tratarem de formas abstratas e também como forma de preparar as crianças para as tarefas posteriores de ordenação com dois pares de estímulos. Todos os participantes foram capazes de responder regularmente.

Sigurdardottir, Green e Saunders (1990) ampliaram esses resultados para três classes de estímulos. O estudo buscou avaliar diferentes modalidades de instruções verbais na formação de classes de estímulos equivalentes, a partir do ensino de relações condicionais e quatro seqüências diferentes com estímulos arbitrários. Os autores sugeriram que os dados de Lazar e Kotlarchyk (1986) poderiam estar equivocados, pois os participantes poderiam estar respondendo aos pares de estímulos por exclusão com base no número limitado de dois pares de estímulos. Em resumo, além da transferência de funções condicionais e ordinais para novos estímulos, as instruções mínimas foram suficientes para estabelecer quatro novas classes ordinais.
Para explicitar esta questão, Stromer e Mackay (1992a) conduziram uma investigação para verificar se o controle condicional é possível sem a necessidade do estabelecimento de pré-requisitos para classes de equivalência via treino de emparelhamento de acordo com o modelo.

Seis crianças com idade variando de 3 a 8 anos participaram desse estudo. Durante o treino inicial um procedimento de esvaecimento foi usado. Com instruções verbais durante o primeiro treino, foi ensinado A $1 \rightarrow$ A 2 ; depois, gradativamente, foram acrescentados os outros estímulos do conjunto "A": $\mathrm{A} 1 \rightarrow \mathrm{A} 2 \rightarrow \mathrm{A} 3, \quad$ A $1 \rightarrow \mathrm{A} 2 \rightarrow \mathrm{A} 3 \rightarrow \mathrm{A} 4$ e A $1 \rightarrow$ A $2 \rightarrow$ A $3 \rightarrow$ A $4 \rightarrow$ A 5 . Cada estímulo adicional foi introduzido em quatro passos de intensidade diferente que variavam do mais visível para o completamente escuro. Cada criança avançaria para o próximo passo de intensidade diferente após ter respondido corretamente em duas tentativas consecutivas sem erro. Os critérios de acerto para avançar para o estágio de treino seguinte eram quatro tentativas corretas consecutivas no passo de esvaecer final. Quando o quinto estímulo era acrescentado, um teste era conduzido com trinta tentativas. Quando a uma palavra sem sentido (BIF) era apresentada, tocar os estímulos na ordem crescente (ou seja, A1 $\rightarrow$ A $2 \rightarrow$ A $3 \rightarrow$ A $4 \rightarrow$ A5) era reforçado e quando uma outra palavra (NUK) era apresentada, tocar os estímulos na ordem decrescente (ou seja, A5 $\rightarrow \mathrm{A} 4 \rightarrow \mathrm{A} 3 \rightarrow \mathrm{A} 2 \rightarrow \mathrm{A} 1)$ era reforçado socialmente.

Os resultados sugerem que o treino independente de conjuntos de estímulos pode estabelecer classes de estímulos sob controle condicional com palavras sem sentido (BIF e NUK) e demonstraram que as palavras exerceram controle condicional sobre o responder ordinal: se BIF, os estímulos deveriam ser tocados em ordem crescente, se NUK, deveriam ser tocados na ordem decrescente. Essas funções se transferiram para o conjunto de estímulos "B" sem treino prévio, e quatro das cinco crianças apresentaram múltipla substituição dos conjuntos originais sob controle condicional das palavras (por exemplo: se BIF, A $1 \rightarrow$ B $2 \rightarrow$ A $3 \rightarrow$ B $4 \rightarrow$ A 5 ; se NUK, A $5 \rightarrow$ B $4 \rightarrow$ A $3 \rightarrow$ B $2 \rightarrow$ A 1 ). Os resultados ampliaram a formação de novas classes ordinais não ensinadas diretamente.

Em um estudo posterior conduzido por Stromer e Mackay (1992b), os autores buscaram avaliar a produção de novas classes de ordem com uma criança normal de dez anos de idade que tinha participado do primeiro estudo. Sua tarefa era selecionar um novo estímulo na posição serial ocupada previamente por um outro estímulo durante o treino. O objetivo deste estudo foi avaliar a mesma relação ordinal apresenta- 
da no experimento anterior dos autores (Stromer \& Mackay, 1992b), substituindo alguns estímulos por outros envolvidos no treino original. O refinamento no procedimento permitiu um resultado significativo na produção de novas classes ordinais a partir desse tipo de treino com MTS. Os autores partiram da noção de que "procedimentos de produção dessas classes geralmente envolvem a exposição de dois ou mais estímulos visuais fisicamente diferentes e contingências de reforçamento que requerem uma ordem específica de respostas a esses estímulos, independente de sua localização espacial" "2. Como resultados, esses autores notaram que as substituições de estímulos realizadas pela criança não variaram. A tendência para apontar estímulos em uma posição particular em uma classe ordinal é remanescente de pesquisas anteriores realizadas por esses autores, em que os participantes respondiam condicionalmente em procedimentos de emparelhamento de acordo com o modelo.

Buscando refinar esses procedimentos de ensino por encadeamento, dois estudos foram programados: no primeiro pretendeu-se replicar sistematicamente os resultados obtidos por Sampaio e Assis (no prelo) com crianças em fase de alfabetização. No segundo estudo, refinou-se o procedimento de ensino adotado por Stromer e Mackay (1992a) com treino por encadeamento introduzindo duas cores (verde e vermelho) como estímulos condicionais. Avaliou-se ainda se os estímulos envolvidos apresentariam as propriedades definidoras de relações ordinais.

\section{ESTUDO 1}

\section{Objetivo}

O Estudo I buscou replicar os resultados obtidos por Sampaio e Assis (no prelo) com crianças normais, avaliando a emergência de classes ordinais, após treino com quatro conjuntos de estímulos diferentes não usuais, através de treino por encadeamento de respostas motoras.

\section{Participantes}

\section{MÉTODO}

Participaram do Estudo 1 seis crianças na faixa etária de 7 anos e 4 meses a 9 anos e 5 meses de idade, cursando a segunda série do ensino fundamental. Os participantes foram recrutados através de contatos pessoais e os seus responsáveis foram informados de que se tratava de uma pesquisa sobre métodos de ensino e que os mesmos receberiam "brindes", material escolar ou um lanche ao final de sua participação em cada sessão experimental, independentemente do seu desempenho. Conforme a Resolução 196/96 do Conselho Nacional de Saúde, todos os responsáveis assinaram um Termo de Compromisso autorizando a participação das crianças.

\section{Ambiente Experimental e Equipamento}

As sessões experimentais foram realizadas em uma sala medindo $6 \mathrm{~m}^{2}$ aproximadamente no Laboratório de Psicologia. O participante sentava-se em frente a um microcomputador, permanecendo o experimentador sentado atrás, monitorando a sessão experimental.

Um microcomputador (modelo IBM Pentium de $300 \mathrm{MHz}$ ) com monitor SVGA de 14' apresentava os estímulos e controlava o número de tentativas e as posições que cada estímulo ocuparam na tela, além do registro das respostas corretas e incorretas. O programa (REL 3.0 for windows) foi elaborado em linguagem Visual Basic 6.0, desenvolvido especialmente para esta pesquisa. Cada sessão experimental teve uma duração de 15 e 20 minutos, no máximo.

\section{Procedimento}

Nas sessões experimentais foram utilizados quatro conjuntos de estímulos, todos não usuais (conforme ilustrado na Figura 1), indicados pelas letras "A" (A1, A2, A3, A4, A5), "B" (B1, B2, B3, B4, B5), "C" (C1, C2, C3, C4, C5) e "D" (D1, D2, D3, D4, D5). 


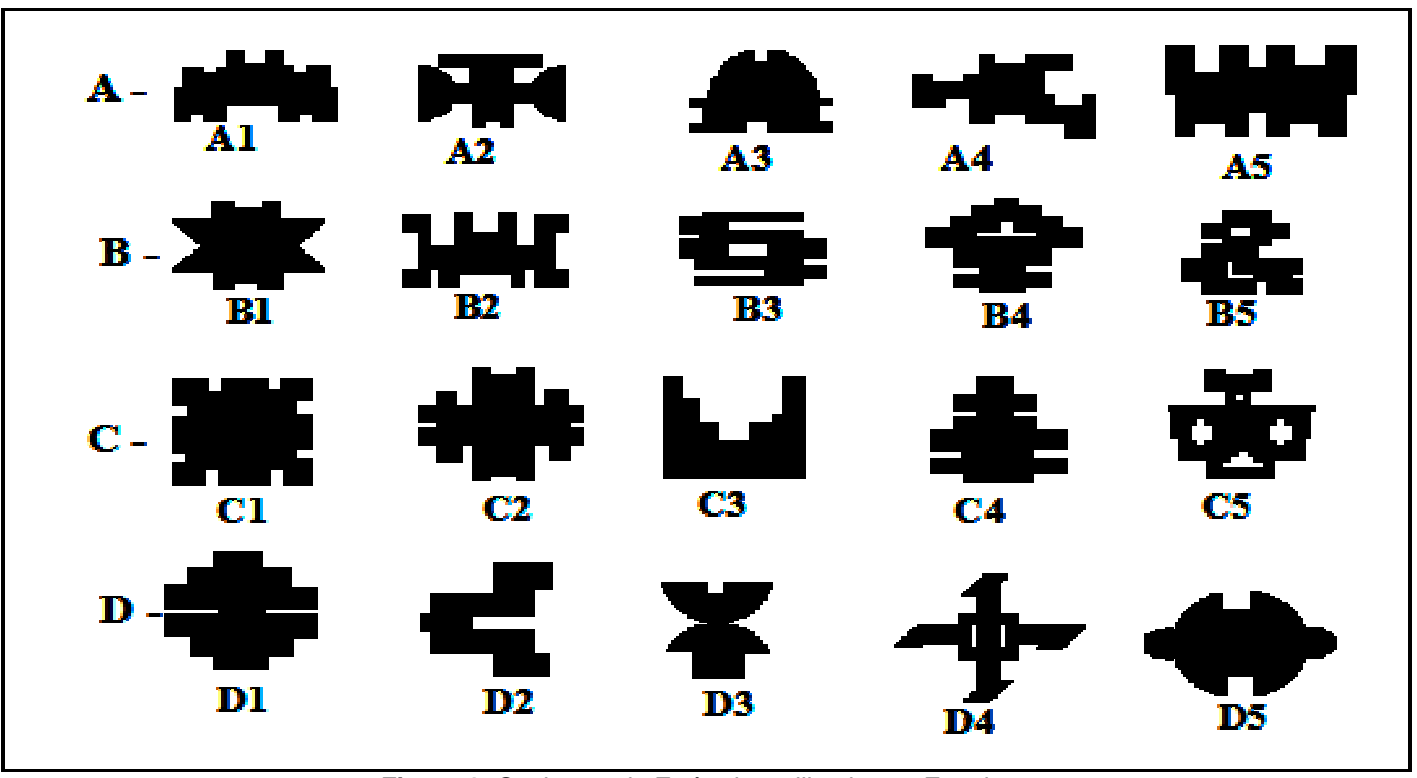

Figura 1: Conjuntos de Estímulos utilizados no Estudo 1.

Os estímulos foram apresentados na tela do computador, que era dividida em duas áreas principais, além de um quadrado na cor cinza, medindo aproximadamente $3 \mathrm{~cm} \times 3 \mathrm{~cm}$, localizado na parte superior da tela, onde se lê a palavra "toque". Logo abaixo do quadrado cinza estava localizada a área, denominada "área de construção", com fundo branco, na qual foram apresentados os estímulos, dispostos lado a lado após desaparecerem da "área de escolha". A segunda área, denominada "área de escolha", também com fundo branco, estava localizada na parte inferior da tela. Aqui o programa reservou oito células de $5,5 \mathrm{~cm}$ $\mathrm{x} 5,5 \mathrm{~cm}$ nas quais apareciam, de forma randomizada, os estímulos que deveriam compor a seqüência. A Tabela 1 apresenta o delineamento experimental previsto para este estudo.

Tabela 1: Sumário dos procedimentos nas fases de treino e testes, tipo e número de tentativa, critério de acerto e probabilidade de reforços no Estudo I

\begin{tabular}{|c|c|c|c|c|c|}
\hline Fase & Bloco de Treino & BLOCO DE TESTE & Tipo de tentativa & $\begin{array}{c}\text { Critério de } \\
\text { Acerto }\end{array}$ & Probabilidade de Reforço \\
\hline & & & $\mathrm{A} 1 \rightarrow \mathrm{A} 2$ & & \\
\hline \multirow[t]{3}{*}{1} & \multirow{3}{*}{$\begin{array}{l}\text { Encadeamento com } \\
\text { conjunto de estímulos A }\end{array}$} & & $\mathrm{A} 1 \rightarrow \mathrm{A} 2 \rightarrow \mathrm{A} 3$ & $3 / 3$ & $1 / 1$ \\
\hline & & & $\mathrm{A} 1 \rightarrow \mathrm{A} 2 \rightarrow \mathrm{A} 3 \rightarrow \mathrm{A} 4$ & & \\
\hline & & & $\mathrm{A} 1 \rightarrow \mathrm{A} 2 \rightarrow \mathrm{A} 3 \rightarrow \mathrm{A} 4 \rightarrow \mathrm{A} 5$ & & \\
\hline 2 & & $\begin{array}{l}\text { Sequenciação com } \\
\text { conjunto de estímulos A }\end{array}$ & $\mathrm{A} 1 \rightarrow \mathrm{A} 2 \rightarrow \mathrm{A} 3 \rightarrow \mathrm{A} 4 \rightarrow \mathrm{A} 5$ & - & \\
\hline \multirow{6}{*}{3} & & \multirow{6}{*}{$\begin{array}{l}\text { Pares de estímulos não } \\
\text { adjacentes }\end{array}$} & $\mathrm{A} 1 \rightarrow \mathrm{A} 3$ & & \\
\hline & & & $\mathrm{A} 1 \rightarrow \mathrm{A} 4$ & & \\
\hline & & & $\mathrm{A} 1 \rightarrow \mathrm{A} 5$ & & \\
\hline & & & $\mathrm{A} 2 \rightarrow \mathrm{A} 4$ & - & \\
\hline & & & $\mathrm{A} 2 \rightarrow \mathrm{A} 5$ & & \\
\hline & & & $\mathrm{A} 3 \rightarrow \mathrm{A} 5$ & & \\
\hline \multirow{4}{*}{4} & Encadeamento com & & $\mathrm{B} 1 \rightarrow \mathrm{B} 2$ & & \\
\hline & conjunto de estímulos B & & $\mathrm{B} 1 \rightarrow \mathrm{B} 2 \rightarrow \mathrm{B} 3$ & & \\
\hline & & & $\mathrm{B} 1 \rightarrow \mathrm{B} 2 \rightarrow \mathrm{B} 3 \rightarrow \mathrm{B} 4$ & $3 / 3$ & $1 / 1$ \\
\hline & & & $\mathrm{B} 1 \rightarrow \mathrm{B} 2 \rightarrow \mathrm{B} 3 \rightarrow \mathrm{B} 4 \rightarrow \mathrm{B} 5$ & & \\
\hline
\end{tabular}




\begin{tabular}{|c|c|c|c|c|c|}
\hline 5 & & conjunto de estímulos B & $\mathrm{B} 1 \rightarrow \mathrm{B} 2 \rightarrow \mathrm{B} 3 \rightarrow \mathrm{B} 4 \rightarrow \mathrm{B} 5$ & - & \\
\hline \multirow{6}{*}{6} & & & $\mathrm{~B} 1 \rightarrow \mathrm{B} 3$ & & \\
\hline & & Pares de estímulos não & $\mathrm{B} 1 \rightarrow \mathrm{B} 4$ & & \\
\hline & & adjacentes & $\mathrm{B} 1 \rightarrow \mathrm{B} 5$ & & \\
\hline & & & $\mathrm{B} 2 \rightarrow \mathrm{B} 4$ & - & \\
\hline & & & $\mathrm{B} 2 \rightarrow \mathrm{B} 5$ & & \\
\hline & & & $\mathrm{B} 3 \rightarrow \mathrm{B} 5$ & & \\
\hline \multirow[b]{2}{*}{7} & Revisão de linha de base & & & & \\
\hline & $\begin{array}{l}\text { com os conjuntos de } \\
\text { estímulos } \mathrm{A} \text { e B }\end{array}$ & & $\begin{array}{l}\mathrm{A} 1 \rightarrow \mathrm{A} 2 \rightarrow \mathrm{A} 3 \rightarrow \mathrm{A} 4 \rightarrow \mathrm{A} 5 \\
\mathrm{~B} 1 \rightarrow \mathrm{B} 2 \rightarrow \mathrm{B} 3 \rightarrow \mathrm{B} 4 \rightarrow \mathrm{B} 5\end{array}$ & $3 / 3$ & \\
\hline \multirow{3}{*}{8} & & & $\mathrm{~A} 1 \rightarrow \mathrm{B} 2 \rightarrow \mathrm{A} 3 \rightarrow \mathrm{B} 4 \rightarrow \mathrm{A} 5$ & & \\
\hline & & Substitutabilidade & $\mathrm{B} 1 \rightarrow \mathrm{A} 2 \rightarrow \mathrm{B} 3 \rightarrow \mathrm{A} 4 \rightarrow \mathrm{B} 5$ & - & \\
\hline & & & $\mathrm{C} 1 \rightarrow \mathrm{C} 2$ & & \\
\hline \multirow{3}{*}{9} & Encadeamento com & & $\mathrm{C} 1 \rightarrow \mathrm{C} 2 \rightarrow \mathrm{C} 3$ & & \\
\hline & conjunto de estímulos C & & $\mathrm{C} 1 \rightarrow \mathrm{C} 2 \rightarrow \mathrm{C} 3 \rightarrow \mathrm{C} 4$ & $3 / 3$ & $1 / 1$ \\
\hline & & & $\mathrm{C} 1 \rightarrow \mathrm{C} 2 \rightarrow \mathrm{C} 3 \rightarrow \mathrm{C} 4 \rightarrow \mathrm{C} 5$ & & \\
\hline \multirow[t]{3}{*}{10} & & $\begin{array}{c}\text { Sequenciação com } \\
\text { conjunto de estímulos C }\end{array}$ & $\mathrm{C} 1 \rightarrow \mathrm{C} 2 \rightarrow \mathrm{C} 3 \rightarrow \mathrm{C} 4 \rightarrow \mathrm{C} 5$ & - & \\
\hline & & & $\mathrm{C} 1 \rightarrow \mathrm{C} 3$ & & \\
\hline & & & $\mathrm{C} 1 \rightarrow \mathrm{C} 4$ & & \\
\hline \multirow[t]{5}{*}{11} & & Pares de estímulos não & $\mathrm{C} 1 \rightarrow \mathrm{C} 5$ & - & \\
\hline & & adjacentes & $\mathrm{C} 2 \rightarrow \mathrm{C} 4$ & & \\
\hline & & & $\mathrm{C} 2 \rightarrow \mathrm{C} 5$ & & \\
\hline & & & $\mathrm{C} 3 \rightarrow \mathrm{C} 5$ & & \\
\hline & & & $\mathrm{D} 1 \rightarrow \mathrm{D} 2$ & & \\
\hline \multirow{3}{*}{12} & Encadeamento com & & $\mathrm{D} 1 \rightarrow \mathrm{D} 2 \rightarrow \mathrm{D} 3$ & & \\
\hline & conjunto de estímulos $\mathrm{D}$ & & $\mathrm{D} 1 \rightarrow \mathrm{D} 2 \rightarrow \mathrm{D} 3 \rightarrow \mathrm{D} 4$ & $3 / 3$ & $1 / 1$ \\
\hline & & & $\mathrm{D} 1 \rightarrow \mathrm{D} 2 \rightarrow \mathrm{D} 3 \rightarrow \mathrm{D} 4 \rightarrow \mathrm{D} 5$ & & \\
\hline \multirow[t]{4}{*}{13} & & $\begin{array}{c}\text { Sequenciação com } \\
\text { conjunto de estímulos D }\end{array}$ & $\mathrm{D} 1 \rightarrow \mathrm{D} 2 \rightarrow \mathrm{D} 3 \rightarrow \mathrm{D} 4 \rightarrow \mathrm{D} 5$ & - & \\
\hline & & & $\mathrm{D} 1 \rightarrow \mathrm{D} 3$ & & \\
\hline & & Pares de estímulos não & $\mathrm{D} 1 \rightarrow \mathrm{D} 4$ & & \\
\hline & & adjacentes & $\mathrm{D} 1 \rightarrow \mathrm{D} 5$ & & \\
\hline \multirow[t]{4}{*}{14} & & & $\mathrm{D} 2 \rightarrow \mathrm{D} 4$ & - & \\
\hline & & & $\mathrm{D} 2 \rightarrow \mathrm{D} 5$ & & \\
\hline & & & D3 $\rightarrow$ D5 & & \\
\hline & & & $\mathrm{C} 1 \rightarrow \mathrm{D} 2 \rightarrow \mathrm{C} 3 \rightarrow \mathrm{D} 4 \rightarrow \mathrm{C} 5$ & - & \\
\hline 15 & & Substitutabilidade & $\mathrm{D} 1 \rightarrow \mathrm{C} 2 \rightarrow \mathrm{D} 3 \rightarrow \mathrm{C} 4 \rightarrow \mathrm{D} 5$ & & \\
\hline
\end{tabular}

Pré-testes: Com objetivo de avaliar algumas propriedades relacionais abstratas, por exemplo: acima, abaixo, próximo, distante, ao lado de, maior que, menor que (cf. Catania, 1998/1999, p. 261-262) envolvidas no tatear (Skinner, 1957/1992), utilizou-se do Instrumento de Avaliação de Repertório Básico para Alfabetização (IAR-Sérgio Leite, 1984). Em seguida, todos os participantes foram expostos a um pré-treino para familiarizar-se com o equipamento e o procedimento de ensino.

Fase 1: Nesta fase foi realizado um treino por encadeamento de respostas com o conjunto de estímulos "A". O experimentador forneceu a seguinte instrução ao participante:

"Veja o quadrado que aparece na parte superior da tela. [o experimentador aponta o quadrado]. Toque no quadrado. Agora aparece uma figura na parte inferior da tela [o experimentador aponta a parte inferior da tela]. Toque na figura. Agora você ouvirá aplausos e verá uma mensagem escrita na tela "muito bem, você acertou!". Entendeu? então, toque no quadrado”.

PAUSA
"Toque novamente no quadrado; agora aparecem duas figuras na parte inferior da tela. Agora, toque primeiro em uma das figuras e depois na outra, depois que você tocar na figura, ela vai desaparecer $e$ reaparecer na parte superior da tela. [O experimentador aponta as figuras]. Entendeu? Então, toque no quadrado".

Inicialmente, uma única figura era apresentada na "janela" medindo aproximadamente $2,5 \mathrm{~cm}$ x $2,5 \mathrm{~cm}$ que estava disposta na "área de escolha". Na primeira tentativa, o estímulo A1, por exemplo, era apresentado no interior dessa "janela", enquanto as demais permaneciam desativadas. Um toque na figura produzia seu deslocamento da "área de escolha" para a "área de construção". Após a resposta do participante, uma animação gráfica aparecia na tela do computador juntamente com o som "Muito bem, você acertou", ou "Parabéns". Em seguida, uma outra figura era adicionada, por exemplo, A1 e A2. A tarefa do participante era responder a $\mathrm{A} 1$ e em seguida a A2; após essa resposta, os estímulos se deslocavam da "área de escolha" para a "área de construção", um de cada vez da esquerda para a direita na parte superior da tela (área 
de construção, ver Figura 3). Em seguida, uma animação gráfica aparecia na tela do computador com a seguinte mensagem sonora: "Muito bem, você acertou", ou "Parabéns". O participante era exposto a cada sequiência correta até três vezes consecutivas, sem erro. Após alcançar esse critério de acerto para a formação das sequiências, uma outra figura era adicionada, por exemplo, A1, A2 e A3, e assim sucessivamente, com cada figura sendo introduzida gradativamente nesta seqüência: A $1 \rightarrow \mathrm{A} 2 \rightarrow \mathrm{A} 3 \rightarrow \mathrm{A} 4 \rightarrow \mathrm{A} 5$.

Quando a resposta era incorreta (por exemplo, $\mathrm{A} 2 \rightarrow \mathrm{A} 1 \rightarrow \mathrm{A} 3)$, não havia consequiência reforçadora, a tela se embranquecia por um intervalo de $1 \mathrm{~s}$ e os mesmos estímulos reapareciam na "área de escolha" em uma outra posição. O participante era exposto à mesma seqüência até 10 vezes, no máximo. Caso o participante continuasse a não formar a seqüência prevista, não era exposto ao teste de seqüenciação, sendo exposto ao mesmo treino com outro conjunto de estímulos. Após alcançar o critério de acerto na última sequiência de treino prevista, o participante era exposto aos testes de seqüenciação e de pares de estímulos não-adjacentes.

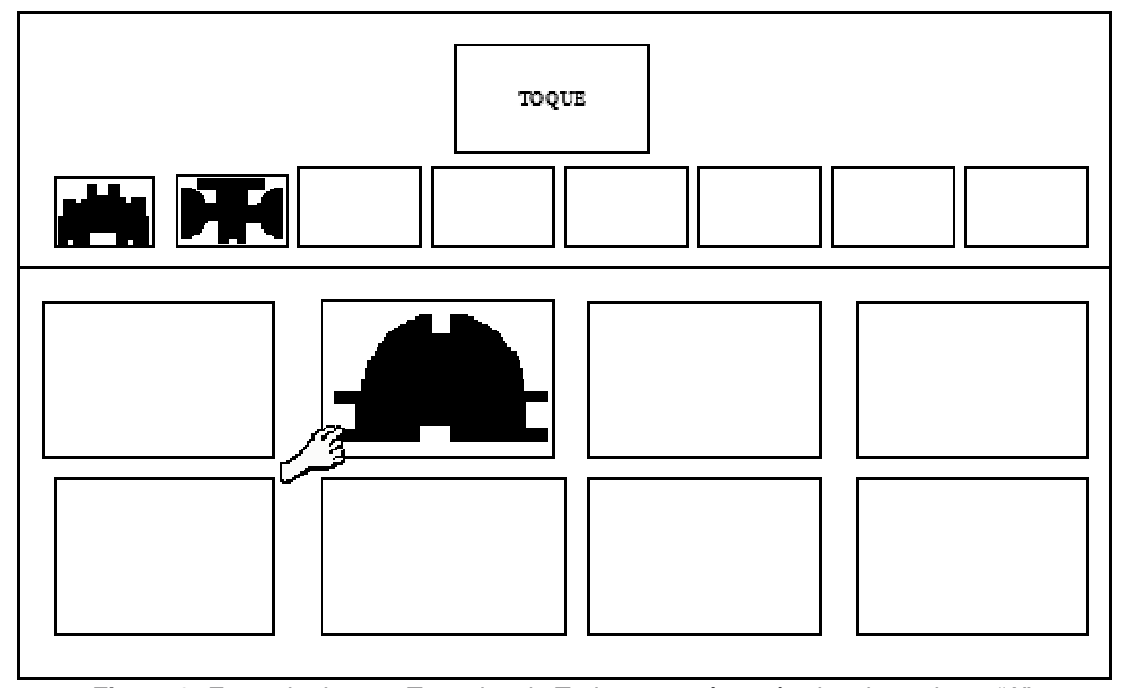

Figura 2: Exemplo de uma Tentativa de Treino com três estímulos do conjunto "A".

Fase 2: Nesta fase o participante era submetido ao teste de seqüenciação. Todos os estímulos do conjunto "A" eram apresentados em "janelas" dispostas lado a lado, simultaneamente, na "área de escolha". O experimentador dizia ao participante:

"Você deverá tocar no quadrado. Agora aparecerão cinco figuras na parte inferior da tela. Você deverá tocar uma figura de cada vez. Agora não haverá aplausos e nem aparecerá a mensagem escrita, mas o computador continuará gravando as suas respostas. Você entendeu?".

Na primeira tentativa, todos os estímulos apareciam na "área de escolha" da tela do computador. O participante tinha que tocar os estímulos na ordem treinada anteriormente até que nenhum mais restasse na "área de escolha".

A tarefa do participante era tocar cada estímulo e formar uma ordem correta, como programada pelo experimentador. Cada vez que o participante tocava uma figura, esta desaparecia da "área de escolha" e aparecia na "área de construção" e as demais figuras que restavam na "área de escolha" modificavam sua posição, independentemente de a resposta do participante ter sido correta ou incorreta. Este teste previa uma precisão de $100 \%$. Caso não respondesse corretamente, o participante teria mais uma oportunidade. Este teste teve como objetivo verificar a efetividade do treino utilizado avaliando relações ordinais. 


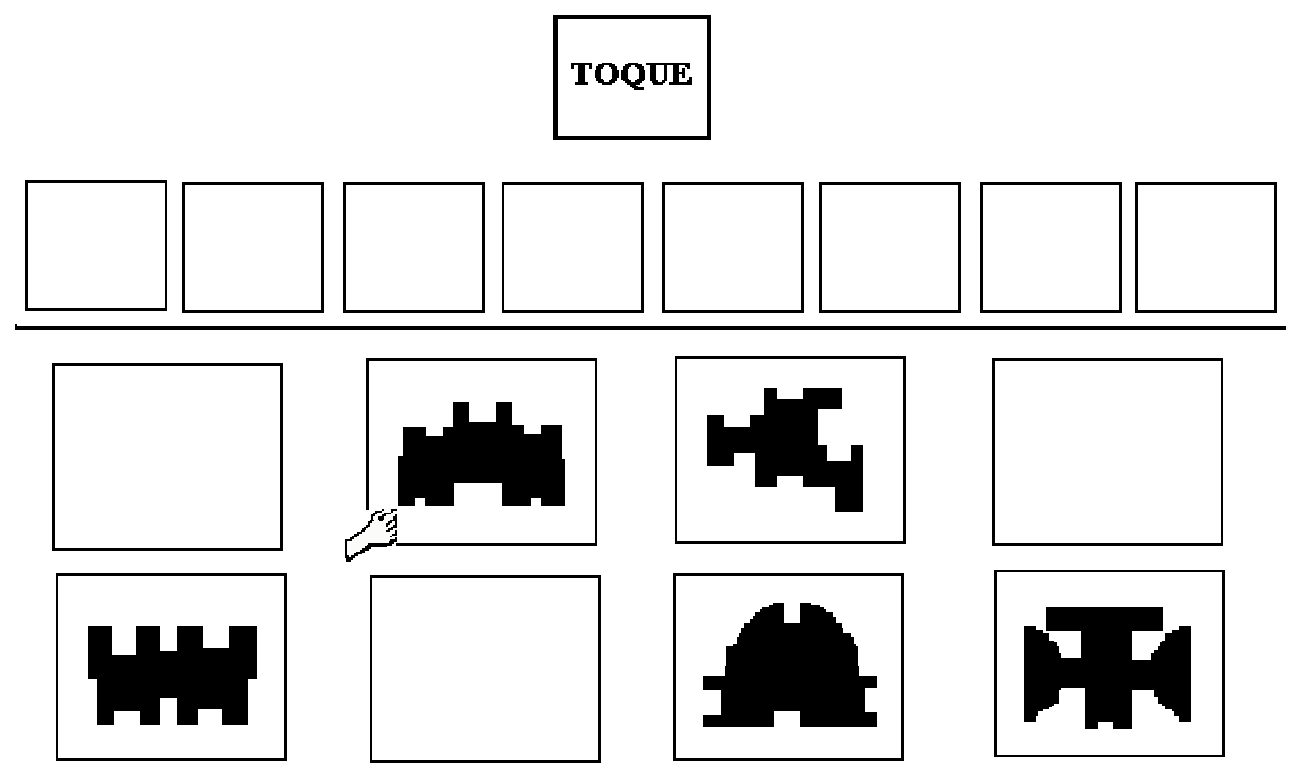

Figura 3: Exemplo de uma Tentativa de Teste de Seqüenciação com estímulos do conjunto "A".

Na Fase 3 foi realizado um teste com pares de estímulos não-adjacentes. No início do teste, o experimentador fornecia a seguinte instrução ao participante:

"Você deverá tocar no quadrado. Aparecerão duas figuras na parte inferior da tela. Você deverá tocar numa e depois na outra. Não haverá aplausos e nem aparecerá a mensagem escrita, mas o computador continuará gravando as suas respostas. Você entendeu?".

Os estímulos do conjunto "A" eram apresentados na "área de escolha" aos pares, em cada tentativa; inicialmente foram apresentados os estímulos A1 e A3. O participante tinha que tocar primeiramente A1, este desaparecia da "área de escolha" e reaparecia na "área de construção" e, em seguida, tocava A3, que também desaparecia da "área de escolha" e reaparecia na "área de construção".
Quando o participante respondia corretamente neste bloco de tentativas, um novo bloco com outro par de estímulos (A1 e A4) era apresentado. A tarefa do participante era tocar primeiramente em A1 e em seguida em A4 e assim sucessivamente, até que todos os pares de estímulos não adjacentes fossem apresentados: A1 $\rightarrow$ A3; A1 $\rightarrow$ A4; A1 $\rightarrow$ A5; A2 $\rightarrow$ A $4 ; \mathrm{A} 2 \rightarrow$ A5 e A3 $\rightarrow$ A5.

Os testes requeriam uma precisão de $100 \%$. Quando o participante respondia corretamente em todas as tentativas, o teste era encerrado e o participante era exposto a um outro bloco de tentativas com um novo par de estímulos não adjacentes. Quando o participante não respondia na sequiência prevista, ele era reexposto a mesma tentativa.

\begin{tabular}{|l|l|l|l|}
\hline $\mathrm{A} 1 \rightarrow \mathrm{A} 2 \rightarrow \mathrm{A} 3 \rightarrow \mathrm{A} 4 \rightarrow \mathrm{A} 5$ \\
\hline $\mathrm{A} 1 \rightarrow \mathrm{A} 2$ & $\mathrm{~A} 1 \rightarrow \mathrm{A} 3$ & $\mathrm{~A} 1 \rightarrow \mathrm{A} 4$ & $\mathrm{~A} 1 \rightarrow \mathrm{A} 5$ \\
\cline { 2 - 4 } & $\mathrm{A} 2 \rightarrow \mathrm{A} 3$ & A2 $\rightarrow$ A4 & A2 $\rightarrow$ A5 \\
\cline { 2 - 4 } & & A3 $\rightarrow$ A4 & A3 $\rightarrow$ A5 \\
\cline { 2 - 4 } & & A4 $\rightarrow$ A5 \\
\cline { 3 - 4 }
\end{tabular}

\section{PARES ADJACENTES}

\section{PARES NÃO ADJACENTES}

Figura 4: Diagrama com pares de estímulos do conjunto A adjacentes e não-adjacentes.

Após o teste de pares de estímulos não-adjacentes de "A", o mesmo procedimento de treino e testes rea- lizado com estímulos do conjunto "A" foi realizado com os estímulos do conjunto B. 
Fase 4: Treino de encadeamento com estímulos do conjunto B;

Fase 5: 'Teste de seqüenciação com estímulos do conjunto B;

Fase 6: Teste com pares de estímulos não-adjacentes do conjunto B;

Fase 7: Após os testes de seqüenciação e com pares de estímulos não-adjacentes do conjunto $\mathrm{B}$, foi realizada uma revisão da linha de base com os estímulos dos conjuntos "A" e "B";

Fase 8: Teste de substitutabilidade: Esta fase previa a exposição ao teste de substitutabilidade para os estímulos das duas sequiências ensinadas: $\mathrm{A} 1 \rightarrow \mathrm{A} 2 \rightarrow \mathrm{A} 3 \rightarrow \mathrm{A} 4 \rightarrow \mathrm{A} 5$ e $\mathrm{B} 1 \rightarrow \mathrm{B} 2 \rightarrow \mathrm{B} 3 \rightarrow \mathrm{B} 4 \rightarrow \mathrm{B} 5$ (ver Figura 5).
Durante esse teste, as seqüências ensinadas " $\mathrm{A}$ " e "B" eram misturadas, por exemplo: $\mathrm{A} 1 \rightarrow \mathrm{B} 2 \rightarrow \mathrm{A} 3 \rightarrow$ $\mathrm{B} 4 \rightarrow \mathrm{A} 5$, em janelas dispostas lado a lado. Todos os estímulos estavam presentes na "área de escolha" da tela do computador e o experimentador dizia ao participante:

"Você deverá tocar no quadrado. Agora aparecerão cinco figuras na parte inferior da tela. Você deverá tocar em uma figura depois da outra. Não haverá aplausos e nem a mensagem escrita, mas o computador continuará gravando as suas respostas. Você entendeu?".

A tarefa do participante era tocar todos os estímulos apresentados até que nenhum mais restasse na "área de escolha". O participante tinha que tocar todos os estímulos que formavam a sequiência numa ordem correta prevista pelo experimentador.

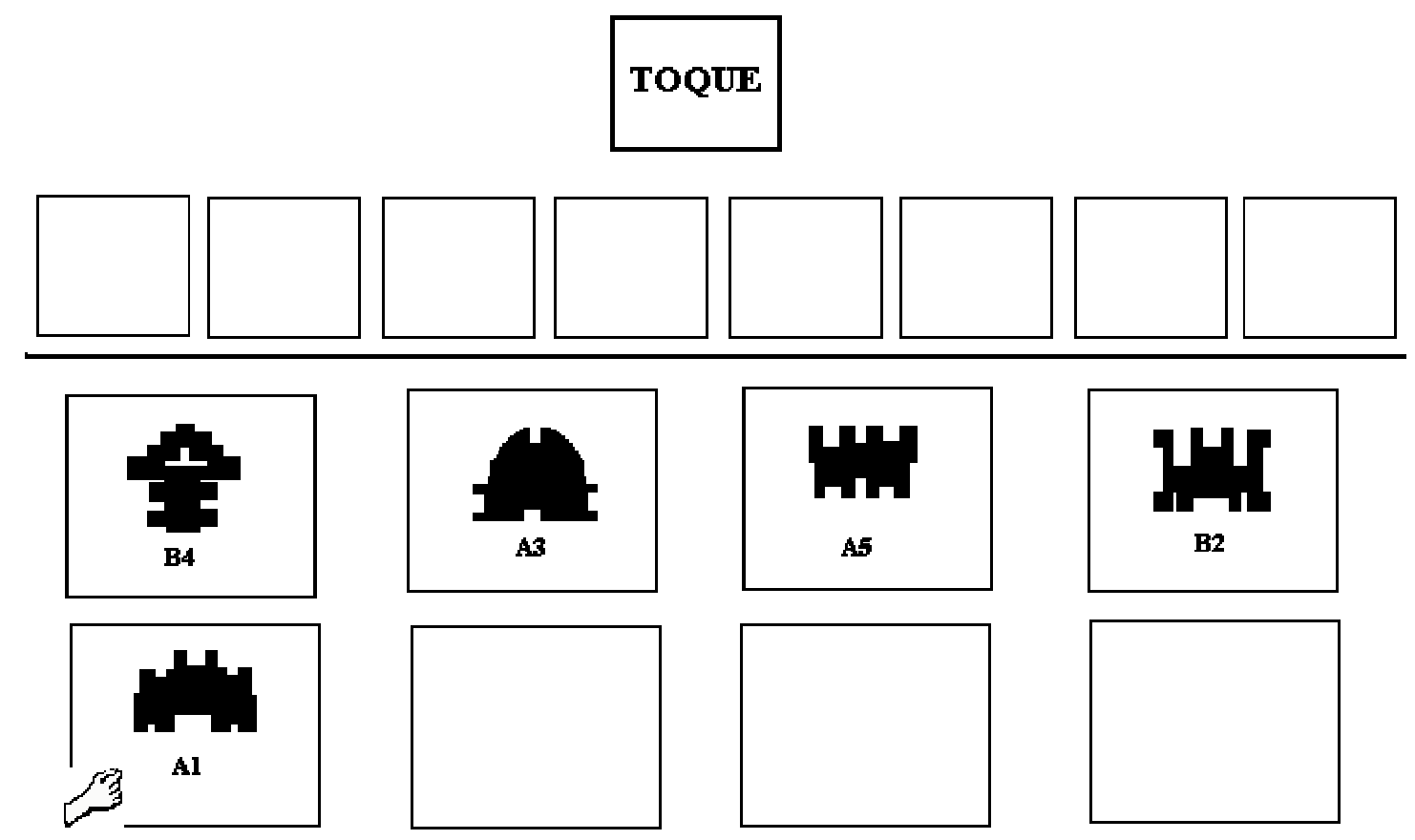

Figura 5 - Exemplo de uma Tentativa de Teste de Substitutabilidade com estímulos dos conjuntos "A" e "B".

Na Fase 09 foi utilizado o mesmo procedimento de treino descrito anteriormente com os estímulos dos conjuntos "A" e "B" para o conjunto de estímulos "C".

Fase 10: Teste de seqüenciação com os estímulos do conjunto "C";

Fase 11: Teste com pares de estímulos não adjacentes com o conjunto "C";
Fase 12: Encadeamento com os estímulos do conjunto "D";

Fase 13: Teste de seqüenciação com os estímulos do conjunto "D";

Fase 14: Teste com pares de estímulos não adjacentes com o conjunto " $D$ "; 
Fase 15: Teste de Substitutabilidade com os estímulos dos conjuntos "C" e "D".

\section{RESULTADOS E DISCUSSÃO}

Resultados dos treinos de encadeamento com os estímulos dos conjuntos "A", "B"," $C$ " $e$ "D"

Seis participantes - NAY, DIE, MGA, KAT, FAB e MAR - foram submetidos aos treinos de encadeamento formados por quatro conjuntos de cinco estímulos não usuais (A, B, C e D). Todos alcançaram a linha de base inicial. Dois (NAY e FAB) responderam prontamente. Os participantes (MGA e MAR) responderam algumas seqüências prontamente. Um participante (KAT) precisou ser reexposto aos treinos com todos os quatro conjuntos de estímulos.

Este treino estabeleceu os pré-requisitos comportamentais para demonstrar que as relações entre os estímulos eram transitivas. Dois participantes (NAY e FAB) responderam prontamente. Os demais precisaram de várias tentativas para alcançar o critério de acerto.

\section{Resultados dos Testes de Seqüenciação com os estímulos dos conjuntos "A", "B", "C" e "D"}

Após os treinos de encadeamento, os participantes foram expostos aos testes de seqüenciação, que previa que cada participante deveria tocar os estímulos apresentados na sequiência anteriormente treinada, até que não restasse nenhum estímulo na "área de escolha". Neste teste, foram programadas duas tentativas: caso o participante respondesse corretamente na primeira tentativa, o teste era encerrado; do contrário, era exposto a uma segunda tentativa.

Três participantes (MGA, KAT e MAR) responderam prontamente aos testes, formando as seqüências com os estímulos dos quatro conjuntos. Os outros três (NAY, DIE e FAB) apresentaram um desempenho parcial nestes testes, respondendo com atraso, ou seja, precisaram de uma segunda tentativa.

\section{Resultados dos testes com pares de estímulos não adjacentes com os estímulos dos conjun- tos "A", "B", "C" e "D"}

Nos testes com pares de estímulos não-adjacentes, cada participante deveria tocar as figuras uma após a outra, definida pelo experimentador. Foram programados seis blocos de teste para cada conjunto de estímulo e cada bloco era formado por duas tentativas; se o participante respondesse corretamente na primeira tentativa, o bloco era encerrado; do contrário ele era exposto a mais uma tentativa.

Todos os seis participantes responderam prontamente aos pares de estímulos não-adjacentes, com os 
quatro conjuntos de estímulos. A regularidade desses resultados parece ocorrer em função da seqüência de testes programada com os pares não-adjacentes. $\mathrm{O}$ arranjo desses testes, a partir do qual podemos inferir relações de transitividade, não foi alternada. Ou seja, o participante sempre iniciava sua exposição com pares não-adjacentes começando com A1, em seguida, as relações iniciadas com A2, depois A3 e assim sucessivamente. A ordem de apresentação das tentativas de testes pode ser uma variável importante, permitindo que o participante responda por exclusão, ou seja, selecionando o primeiro estímulo, não importando qual o segundo disponível na tela.

\section{Resultados da revisão de Linha de Base com os estímulos dos conjuntos "A", "B", "C" e "D"}

Após responderem aos pares de estímulos nãoadjacentes, todos os seis participantes foram submetidos à revisão de linha de base. Um participante (MGA) respondeu prontamente todas as sequiências. Os demais precisaram de mais tentativas para alcançar o critério de acerto (ver Tabela 2).

Tabela 2: Desempenho dos participantes em cada seqüência com os estímulos $A, B, C$ e D

\begin{tabular}{lcccccc}
\hline Bloco & NAY & DIE & MGA & KAT & FAB & MAR \\
\hline $\mathrm{A} 1 \rightarrow \mathrm{A} 2 \rightarrow \mathrm{A} 3 \rightarrow \mathrm{A} 4 \rightarrow \mathrm{A} 5$ & + & + & + & + & & + \\
$\mathrm{B} 1 \rightarrow \mathrm{B} 2 \rightarrow \mathrm{B} 3 \rightarrow \mathrm{B} 4 \rightarrow \mathrm{B} 5$ & + & + & + & + & & + \\
$\mathrm{C} 1 \rightarrow \mathrm{C} 2 \rightarrow \mathrm{C} 3 \rightarrow \mathrm{C} 4 \rightarrow \mathrm{C} 5$ & & & + & + & & \\
$\mathrm{D} 1 \rightarrow \mathrm{D} 2 \rightarrow \mathrm{D} 3 \rightarrow \mathrm{D} 4 \rightarrow \mathrm{D} 5$ & & + & + & & + & + \\
\hline
\end{tabular}

+ Respondeu prontamente

O número de tentativas variou, mas o participante alcançou o critério de acerto.

Resultados dos testes de substitutabilidade com os estímulos dos conjuntos "A", "B", "C" e "D"

Todos os participantes foram expostos aos testes de substitutabilidade. Nestes testes cinco estímulos de dois conjuntos ("A" e "B" ou "C" e "D") apareceram randomicamente na área de escolha. A tarefa do participante era tocar os estímulos em uma ordem correta, especificada pelo experimentador. Caso o participante tocasse os estímulos na ordem correta na primeira tentativa, o bloco era finalizado; do contrário, ele era exposto a uma outra tentativa.

Este teste teve o objetivo de analisar a formação de classes de estímulos ordinais derivadas do treino original. Desta forma, poderia ser demonstrada as propriedades de transitividade da relação entre estímulos dentro de ambas as sequiências ensinadas. Para que esta propriedade fosse demonstrada, os estímulos en- tre as seqüências deveriam tornar-se mutuamente substituíveis uns pelos outros.

Os participantes NAY, MGA e KAT responderam prontamente aos testes de substitutabilidade, enquanto que DIE, FAB e MAR precisaram ser reexpostos às seqüências $\mathrm{C} 1 \rightarrow \mathrm{D} 2 \rightarrow \mathrm{C} 3 \rightarrow \mathrm{D} 4 \rightarrow \mathrm{C} 5, \mathrm{~A} 1 \rightarrow \mathrm{B} 2 \rightarrow \mathrm{A} 3 \rightarrow$ $\mathrm{B} 4 \rightarrow \mathrm{A} 5$ e $\mathrm{B} 1 \rightarrow \mathrm{A} 2 \rightarrow \mathrm{B} 3 \rightarrow \mathrm{A} 4 \rightarrow \mathrm{B} 5$.

A partir destes dados pode-se inferir que o treino forneceu base suficiente para a emergência de novas sequiências, já que os participantes produziram as novas sequiências tocando os estímulos em uma ordem que foi consistente com o treino, mesmo que os estímulos nunca tivessem aparecido juntos anteriormente. Aparentemente, os estímulos que ocuparam a mesma posição ordinal em diferentes seqüências vieram a formar classes de estímulos funcionalmente equivalentes.

\begin{tabular}{|c|c|c|c|c|c|c|}
\hline Testes de Substitutabilidade & NAY & DIE & MGA & KAT & FAB & MAR \\
\hline $\mathrm{A} 1 \rightarrow \mathrm{B} 2 \rightarrow \mathrm{A} 3 \rightarrow \mathrm{B} 4 \rightarrow \mathrm{A} 5$ & + & & + & + & & + \\
\hline $\mathrm{B} 1 \rightarrow \mathrm{A} 2 \rightarrow \mathrm{B} 3 \rightarrow \mathrm{A} 4 \rightarrow \mathrm{B} 5$ & + & + & + & + & + & \\
\hline $\mathrm{C} 1 \rightarrow \mathrm{D} 2 \rightarrow \mathrm{C} 3 \rightarrow \mathrm{D} 4 \rightarrow \mathrm{C} 5$ & + & & + & + & + & + \\
\hline $\mathrm{D} 1 \rightarrow \mathrm{C} 2 \rightarrow \mathrm{D} 3 \rightarrow \mathrm{C} 4 \rightarrow \mathrm{D} 5$ & + & + & + & + & + & + \\
\hline
\end{tabular}

+ Respondeu prontamente

Respondeu com atraso.

\section{ESTUDO 2}

\section{Objetivo}

O Estudo 2 pretendeu estender os resultados do Estudo 1 a crianças do pré-escolar, buscando avaliar se esse responder ordinal ainda se manteria estável quando as crianças respondessem sob controle condicional, avaliando, em seguida, se os estímulos envolvidos seriam funcionalmente equivalentes. 


\section{MÉTODO}

\section{Participantes}

Participaram do estudo três crianças com idades variando entre 4 anos e 7 meses e 5 anos e 6 meses, que estavam cursando a educação infantil durante a coleta dos dados. Os participantes foram recrutados através de contatos pessoais e os seus responsáveis foram informados de que se tratava de uma pesquisa sobre métodos de ensino e que os mesmos receberiam "brindes", materiais escolar ou um lanche ao final de rizando a participação do aluno no estudo, conforme exigência da Resolução 196/96 do Conselho Nacional de Saúde.

\section{Ambiente experimental e equipamento}

As condições experimentais e o equipamento foram as mesmas do Estudo 1.

\section{Procedimento}

Foram utilizados dois conjuntos de estímulos, for-

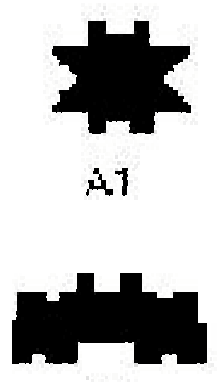

B1

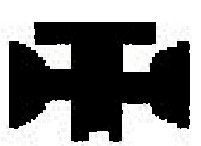

A2

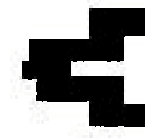

$\mathrm{B2}$

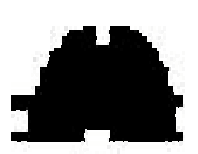

$\Delta 3$

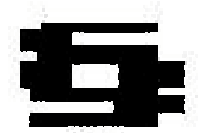

B3

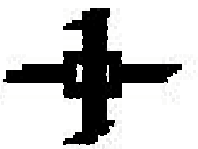

A4

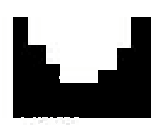

$\mathrm{B} 4$
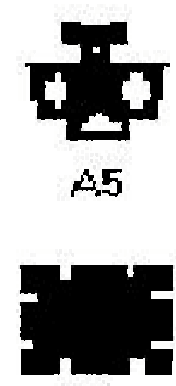

85 sua participação em cada sessão experimental, independente do seu desempenho. Um Termo de Compromisso foi assinado pelos pais ou responsáveis auto- mados por cinco estímulos não usuais (ilustrados na Figura 6). Um conjunto foi identificado pela letra "A" e o outro pela letra "B".

Figura 6: - Conjuntos de Estímulos utilizados no Estudo 2.

Após ensinar a tarefa de ordenar as figuras do conjunto "A" a partir de A1 até A5, uma cor verde foi introduzida na parte superior central da tela. Agora o participante deveria primeiro responder na cor e em seguida ordenar as figuras na mesma direção (A1 até A5). Em seguida, um teste de sequenciação foi aplicado na presença da cor verde. Caso a resposta de ordenar fosse correta, o participante avançaria para o bloco de treino do mesmo conjunto de estímulos ("A"). Agora na presença da cor vermelha que permanecia na parte superior central da tela, o participante foi ensinado a tocar nessa cor e depois ordenar as figuras de A5 até A1. Em seguida, testes de sequenciação foram aplicados para avaliar se o participante continuaria respondendo sem conseqüência diferencial (ver Tabela 4).

A Tabela 4 apresenta um sumário do delineamento experimental com os procedimentos de treino por encadeamento, testes de seqüenciação, testes com pares de estímulos não-adjacentes e de substitutabilidade na presença das cores verde e vermelha com os estímulos dos conjuntos "A" e "B". Além disso, a Tabela 4 apresenta o tipo de tentativa, critério de acerto e probabilidade de reforços após cada tentativa. Ou seja, o procedimento foi o mesmo adotado no Estudo 1 agora com a introdução das cores verde e vermelha como estímulos condicionais.

Tabela 4: Delineamento experimental dos procedimentos de treino por encadeamento, testes de seqüenciação, com pares de estímulos não adjacentes, substitutabilidade e revisão de linha de base com os estímulos dos conjuntos "A" e "B" na presença das cores verde e vermelha

\begin{tabular}{|c|c|c|c|c|c|c|}
\hline Fase & Bloco de Treino & Bloco de Teste & Tipo de tentativa em seqüência & $\begin{array}{l}\text { Estímulo } \\
\text { Condicional } \\
\text { "CORES" }\end{array}$ & $\begin{array}{l}\text { Critério de } \\
\text { Acerto }\end{array}$ & $\begin{array}{c}\text { Probabilidade } \\
\text { de Reforço }\end{array}$ \\
\hline \multirow{4}{*}{1} & Encadeamento & & $\mathrm{A} 1 \rightarrow \mathrm{A} 2$ & & & \\
\hline & \multirow{3}{*}{$\begin{array}{l}\text { com conjunto de } \\
\text { estímulos "A" }\end{array}$} & & $\mathrm{A} 1 \rightarrow \mathrm{A} 2 \rightarrow \mathrm{A} 3$ & Verde & $3 / 3$ & $1 / 1$ \\
\hline & & & $\mathrm{A} 1 \rightarrow \mathrm{A} 2 \rightarrow \mathrm{A} 3 \rightarrow \mathrm{A} 4$ & & & \\
\hline & & & $\mathrm{A} 1 \rightarrow \mathrm{A} 2 \rightarrow \mathrm{A} 3 \rightarrow \mathrm{A} 4 \rightarrow \mathrm{A} 5$ & & & \\
\hline 2 & & $\begin{array}{c}\text { Seqüenciação com } \\
\text { conjunto de estímulos A }\end{array}$ & $\mathrm{A} 1 \rightarrow \mathrm{A} 2 \rightarrow \mathrm{A} 3 \rightarrow \mathrm{A} 4 \rightarrow \mathrm{A} 5$ & Verde & - & \\
\hline
\end{tabular}




\begin{tabular}{|c|c|c|c|c|c|c|}
\hline 3 & $\begin{array}{l}\text { Encadeamento } \\
\text { com conjunto de } \\
\text { estímulos "A" }\end{array}$ & & $\begin{array}{c}\mathrm{A} 5 \rightarrow \mathrm{A} 4 \\
\mathrm{~A} 5 \rightarrow \mathrm{A} 4 \rightarrow \mathrm{A} 3 \\
\mathrm{~A} 5 \rightarrow \mathrm{A} 4 \rightarrow \mathrm{A} 3 \rightarrow \mathrm{A} 2 \\
\mathrm{~A} 5 \rightarrow \mathrm{A} 4 \rightarrow \mathrm{A} 3 \rightarrow \mathrm{A} 2 \rightarrow \mathrm{A} 1\end{array}$ & Vermelho & $3 / 3$ & $1 / 1$ \\
\hline 4 & & $\begin{array}{c}\text { Seqüenciação com } \\
\text { conjunto de estímulos A }\end{array}$ & $\mathrm{A} 5 \rightarrow \mathrm{A} 4 \rightarrow \mathrm{A} 3 \rightarrow \mathrm{A} 2 \rightarrow \mathrm{A} 1$ & Vermelho & - & \\
\hline 5 & $\begin{array}{l}\text { Encadeamento } \\
\text { com conjunto de } \\
\text { estímulos "B" }\end{array}$ & & $\begin{array}{c}\mathrm{B} 1 \rightarrow \mathrm{B} 2 \\
\mathrm{~B} 1 \rightarrow \mathrm{B} 2 \rightarrow \mathrm{B} 3 \\
\mathrm{~B} 1 \rightarrow \mathrm{B} 2 \rightarrow \mathrm{B} 3 \rightarrow \mathrm{B} 4 \\
\mathrm{~B} 1 \rightarrow \mathrm{B} 2 \rightarrow \mathrm{B} 3 \rightarrow \mathrm{B} 4 \rightarrow \mathrm{B} 5\end{array}$ & Verde & $3 / 3$ & $1 / 1$ \\
\hline 6 & & $\begin{array}{l}\text { Teste de Seqüenciação } \\
\text { com estímulos do } \\
\text { Conjunto "B" }\end{array}$ & $\mathrm{B} 1 \rightarrow \mathrm{B} 2 \rightarrow \mathrm{B} 3 \rightarrow \mathrm{B} 4 \rightarrow \mathrm{B} 5$ & Verde & - & \\
\hline 7 & $\begin{array}{l}\text { Encadeamento } \\
\text { com conjunto de } \\
\text { estímulos "B" }\end{array}$ & & $\begin{array}{c}\mathrm{B} 5 \rightarrow \mathrm{B} 4 \\
\mathrm{~B} 5 \rightarrow \mathrm{B} 4 \rightarrow \mathrm{B} 3 \\
\mathrm{~B} 5 \rightarrow \mathrm{B} 4 \rightarrow \mathrm{B} 3 \rightarrow \mathrm{B} 2 \\
\mathrm{~B} 5 \rightarrow \mathrm{B} 4 \rightarrow \mathrm{B} 3 \rightarrow \mathrm{B} 2 \rightarrow \mathrm{B} 1\end{array}$ & Vermelho & $3 / 3$ & $1 / 1$ \\
\hline 8 & & $\begin{array}{l}\text { Teste de Seqüenciação } \\
\text { com conjunto de } \\
\text { estímulos "B" }\end{array}$ & $\mathrm{B} 5 \rightarrow \mathrm{B} 4 \rightarrow \mathrm{B} 3 \rightarrow \mathrm{B} 2 \rightarrow \mathrm{B} 1$ & Vermelho & - & \\
\hline 9 & $\begin{array}{l}\text { Revisão de Linha } \\
\text { de Base com } \\
\text { estímulos dos } \\
\text { conjuntos "A" e "B" }\end{array}$ & & $\begin{array}{l}A 1 \rightarrow A 2 \rightarrow A 3 \rightarrow A 4 \rightarrow A 5 \\
A 5 \rightarrow A 4 \rightarrow A 3 \rightarrow A 2 \rightarrow A 1 \\
B 1 \rightarrow B 2 \rightarrow B 3 \rightarrow B 4 \rightarrow B 5 \\
B 5 \rightarrow B 4 \rightarrow B 3 \rightarrow B 2 \rightarrow B 1\end{array}$ & $\begin{array}{l}\text { Verde } \\
\text { Vermelho } \\
\text { Verde } \\
\text { Vermelho }\end{array}$ & $3 / 3$ & \\
\hline 10 & & $\begin{array}{l}\text { Testes de Substitutabilida- } \\
\text { de com conjuntos de } \\
\text { Estímulos "A" e "B" }\end{array}$ & $\begin{array}{l}A 1 \rightarrow B 2 \rightarrow A 3 \rightarrow B 4 \rightarrow A 5 \\
A 5 \rightarrow B 4 \rightarrow A 3 \rightarrow B 2 \rightarrow A 1 \\
B 1 \rightarrow A 2 \rightarrow B 3 \rightarrow A 4 \rightarrow B 5 \\
B 5 \rightarrow A 4 \rightarrow B 3 \rightarrow A 2 \rightarrow B 1\end{array}$ & $\begin{array}{l}\text { Verde } \\
\text { Vermelho } \\
\text { Verde } \\
\text { Vermelho }\end{array}$ & - & $1 / 1$ \\
\hline
\end{tabular}

\section{RESULTADOS E DISCUSSÃO}

Resultados do Treino por encadeamento na presença das cores "verde" e "vermelha" com os conjuntos de estímulos "A" e "B"

Três participantes, ANA, GAB e MAR foram submetidos a um procedimento de treino por encade- amento com dois conjuntos de estímulos (A e B) na presença das cores verde e vermelha. Cada participante poderia ser exposto a até 10 tentativas, no máximo. Caso alcançasse o critério de acerto (três vezes consecutivas sem erro), avançaria para uma outra seqüência. Um participante (MAR) alcançou prontamente o critério de acerto. Os outros dois (ANA e GAB) precisaram de mais tentativas para alcançar o critério de acerto (ver Tabela 5).

Tabela 5: Desempenho dos participantes, em cada seqüência ensinada com os conjuntos de estímulos $A$ e $B$ na presença das duas cores: verde e vermelho

\begin{tabular}{llccc}
\hline $\begin{array}{l}\text { Estímulo } \\
\text { Condicional }\end{array}$ & Bloco de treino & ANA & GAB & MAR \\
\hline VERDE & $\mathrm{A} 1 \rightarrow \mathrm{A} 2 \rightarrow \mathrm{A} 3 \rightarrow \mathrm{A} 4 \rightarrow \mathrm{A} 5$ & & + & + \\
VERMELHO & $\mathrm{A} 5 \rightarrow \mathrm{A} 4 \rightarrow \mathrm{A} 3 \rightarrow \mathrm{A} 2 \rightarrow \mathrm{A} 1$ & & & + \\
VERDE & $\mathrm{B} 1 \rightarrow \mathrm{B} 2 \rightarrow \mathrm{B} 3 \rightarrow \mathrm{B} 4 \rightarrow \mathrm{B} 5$ & + & + & + \\
VERMELHO & $\mathrm{B} 5 \rightarrow \mathrm{B} 4 \rightarrow \mathrm{B} 3 \rightarrow \mathrm{B} 2 \rightarrow \mathrm{B} 1$ & & & + \\
\hline + Alcançou prontamente o critério de acerto & & & \\
\multicolumn{2}{l}{ O número de tentativas variou, mas o participante alcançou o critério de acerto. }
\end{tabular}

Resultados dos Testes de Seqüenciação na presença das cores "verde" e "vermelha" com os conjuntos de estímulos "A" e "B"
Após o treino por encadeamento, os três participantes foram expostos ao teste de seqüenciação, em extinção, na presença das cores verde ou vermelho. Um participante (ANA) respondeu a todos os testes 
com $100 \%$ de acurácia na primeira tentativa de cada (ver Tabela 6). sequiência. Os demais precisaram de mais tentativas

Tabela 6: Desempenho dos participantes em cada seqüência de estímulos A e B na presença das duas cores: verde e vermelho

\begin{tabular}{llccc}
\hline $\begin{array}{l}\text { Estímulo } \\
\text { Condicional }\end{array}$ & Testes de Sequenciação & ANA & GAB & MAR \\
\hline VERDE & $\mathrm{A} 1 \rightarrow \mathrm{A} 2 \rightarrow \mathrm{A} 3 \rightarrow \mathrm{A} 4 \rightarrow \mathrm{A} 5$ & + & & \\
VERMELHO & $\mathrm{A} 5 \rightarrow \mathrm{A} 4 \rightarrow \mathrm{A} 3 \rightarrow \mathrm{A} 2 \rightarrow \mathrm{A} 1$ & + & & \\
VERDE & $\mathrm{B} 1 \rightarrow \mathrm{B} 2 \rightarrow \mathrm{B} 3 \rightarrow \mathrm{B} 4 \rightarrow \mathrm{B} 5$ & + & + & + \\
VERMELHO & $\mathrm{B} 5 \rightarrow \mathrm{B} 4 \rightarrow \mathrm{B} 3 \rightarrow \mathrm{B} 2 \rightarrow \mathrm{B} 1$ & + & + & + \\
\hline
\end{tabular}

+ Respondeu prontamente

Respondeu com atraso.

Resultados da revisão de Linha de Base na presença das cores "verde" e "vermelha" com os conjuntos de estímulos "A" $\mathrm{e}$ "B"

Todos os participantes responderam prontamente quando foram submetidos à revisão de Linha de Base. Novamente, estes resultados demonstram que o treino por encadeamento de respostas foi efetivo na instalação do comportamento de ordenar formas abstratas. Apenas um participante (GAB) precisou de mais tentativas para alcançar o critério de acerto, como ilustrado na Tabela 7.

Tabela 7: Desempenho dos participantes na linha de base em cada seqüência com os conjuntos de estímulos A e B na presença de duas cores: verde e vermelho

\begin{tabular}{lllll}
\hline $\begin{array}{l}\text { Estímulo } \\
\text { Condicional }\end{array}$ & Bloco & ANA & GAB & MAR \\
\hline VERDE & $\mathrm{A} 1 \rightarrow \mathrm{A} 2 \rightarrow \mathrm{A} 3 \rightarrow \mathrm{A} 4 \rightarrow \mathrm{A} 5$ & + & + & + \\
VERMELHO & $\mathrm{A} 5 \rightarrow \mathrm{A} 4 \rightarrow \mathrm{A} 3 \rightarrow \mathrm{A} 2 \rightarrow \mathrm{A} 1$ & + & + & + \\
VERDE & $\mathrm{B} 1 \rightarrow \mathrm{B} 2 \rightarrow \mathrm{B} 3 \rightarrow \mathrm{B} 4 \rightarrow \mathrm{B} 5$ & + & + & + \\
VERMELHO & $\mathrm{B} 5 \rightarrow \mathrm{B} 4 \rightarrow \mathrm{B} 3 \rightarrow \mathrm{B} 2 \rightarrow \mathrm{B} 1$ & + & & + \\
\hline Respondeu prontamente & & & \\
O número de tentativas variou, mas o participante alcançou o critério de acerto.
\end{tabular}

Resultados dos Testes de Substitutabilidade na presença das cores "verde" $\mathrm{e}$ "vermelha" com os conjuntos de estímulos "A" $\mathrm{e}$ "B"

Após os testes de seqüenciação, todos os participantes foram submetidos à Revisão de Linha de Base e em seguida submetidos aos testes de substitutabilidade. Dois participantes responderam prontamente a todos os testes de substitutabilidade. Um participante (GAB) não respondeu ao Teste que envolvia os estímulos dos conjuntos "A" e "B" na presença da cor "vermelha", ou seja, A5 $\rightarrow \mathrm{B} 4 \rightarrow \mathrm{A} 3 \rightarrow \mathrm{B} 2 \rightarrow \mathrm{A} 1$ (ver
Tabela 8). Deve-se considerar que a quantidade de novas seqüências programada foi limitada. Ou seja, deveriam ter sido planejada primeiramente seqüências com dois estímulos, depois com três e assim sucessivamente até completar a seqüência mais longa com cinco estímulos.

Uma outra possibilidade seria o uso de sondas com tentativas de testes de seqüenciação após cada treino com dois, três, quatro e cinco estímulos, reduzindo assim a probabilidade de erros durante o treino por encadeamento.

Tabela 8: Desempenho dos participantes em cada seqüência mista testada com os conjuntos de estímulos A e B na presença de duas cores: verde e vermelho

\begin{tabular}{lcccc}
\hline Estimulo Condicional & Testes de Substitutabilidade & ANA & GAB & MAR \\
\hline VERDE & $\mathrm{A} 1 \rightarrow \mathrm{B} 2 \rightarrow \mathrm{A} 3 \rightarrow \mathrm{B} 4 \rightarrow \mathrm{A} 5$ & + & + & + \\
VERMELHO & $\mathrm{A} 5 \rightarrow \mathrm{B} 4 \rightarrow \mathrm{A} 3 \rightarrow \mathrm{B} 2 \rightarrow \mathrm{A} 1$ & + & - & + \\
VERDE & $\mathrm{B} 1 \rightarrow \mathrm{A} 2 \rightarrow \mathrm{B} 3 \rightarrow \mathrm{A} 4 \rightarrow \mathrm{B} 5$ & + & & +
\end{tabular}




\begin{tabular}{ccc} 
VERMELHO & $\mathrm{B} 5 \rightarrow \mathrm{A} 4 \rightarrow \mathrm{B} 3 \rightarrow \mathrm{A} 2 \rightarrow \mathrm{B} 1$ & + \\
\hline+ Respondeu prontamente & Respondeu com atraso & - Não respondeu ao teste
\end{tabular}

\section{DISCUSSÃO GERAL}

O presente estudo buscou generalizar os resultados obtidos por Sampaio e Assis (no prelo) no Estudo 1 com discriminações simples e replicar sistematicamente o de Stromer e Mackay (1992a) no Estudo 2 avaliando a emergência de classes ordinais sob controle condicional, ambos com crianças normais. A partir de um treino por encadeamento com pelo menos duas seqüências independentes, o participante precisava ordenar (primeiro, segundo, terceiro, quarto e quinto) cinco estímulos visuais diferentes, conforme uma ordem prevista pelo experimentador. A ênfase deste tipo de análise requer, inicialmente, a avaliação das funções que cada membro ocupa numa classe ordinal e as relações estabelecidas entre os estímulosmembro desta e especialmente entre membros de classes diferentes.

O estudo conduzido por Sampaio e Assis (no prelo) demonstrou que, com portadores de deficiência mental, o procedimento de treino por encadeamento de respostas utilizado para estabelecer classes ordinais independentes foi eficiente e também suficiente para a emergência de novas classes ordinais sem qualquer treino adicional. Os dados do presente estudo replicaram e ampliaram os resultados obtidos por Sampaio e Assis, estendendo-os para uma população de crianças normais. $\mathrm{O}$ arranjo de treino que foi organizado mostrou-se efetivo para o estabelecimento de relações ordinais, sem a necessidade de ensinar diretamente novas seqüências de respostas. A exposição direta dos participantes a estímulos não usuais também diferenciou este estudo daquele apresentado pelos autores acima; e a emergência de classes ordinais, sem a necessidade de treino adicional na presença de estímulos usuais (familiares), nos leva a inferir a ampliação desses resultados.

Os resultados do estudo conduzido por Stromer e Mackay (1993) apontaram que o ensino de relações entre estímulos que ocuparam posições ordinais correspondentes em diferentes classes ordinais ofereceu base suficiente para a emergência de novas classes ordinais. Os participantes produziram novas classes ordinais não diretamente ensinadas em uma ordem que foi consistente com as contingências de reforçamento, mesmo que os estímulos nunca tivessem aparecido juntos anteriormente. Segundo esses autores (Stromer \& Mackay, 1993): "aparentemente, os estímulos que ocuparam a mesma posição ordinal em diferentes conjuntos formaram classes de estímulos funcionalmente equivalentes" (p. 129). Corroborando esses resultados, o presente estudo confirmou este achado experimental, verificando que os estímulos que ocuparam a mesma posição em cada conjunto foram membros de classes de estímulos funcionalmente equivalentes.

De acordo com Stromer e Mackay, (1992b), a transferência de controle condicional para estas novas classes ordinais apóia a noção de que o treino estabeleceu classes de estímulos funcionais. Eles apontaram a possibilidade de se avaliar a transferência de controle condicional de um conjunto para outro, demonstrando que os estímulos condicionais utilizados exerceram controle condicional na formação de novas classes ordinais (A $1 \rightarrow$ B $2 \rightarrow$ A $3 \rightarrow$ B $4 \rightarrow$ A 5 e $\mathrm{B} 1 \rightarrow \mathrm{A} 2 \rightarrow \mathrm{B} 3 \rightarrow \mathrm{A} 4 \rightarrow \mathrm{B} 5)$.

Os resultados apresentados no presente estudo parecem evidenciar que as propriedades das relações equivalentes seriam independentes (Pilgrim \& Galizio, 2000). Todos os participantes responderam prontamente a pares de estímulos não-adjacentes, provavelmente uma relação mais simples. Porém, nos dois estudos alguns participantes responderam com atraso em testes de substitutabilidade (ver Tabelas 3 e 8 ). Este teste exige uma intercambialidade funcional na emergência de novas seqüências. Portanto, relações mais complexas.

Os dados apresentados do segundo estudo estendem esses resultados para crianças do pré-escolar, demonstrando a estabilidade em que se manteve o responder ordinal sob controle condicional. Além disso, as duas cores utilizadas exerceram uma função discriminativa de segunda ordem (cf. Sidman, 1986).

Esses resultados podem ainda contribuir para análise de comportamentos conceituais numéricos e da sintaxe, através da elaboração de procedimentos de ensino que envolva relações ordinais, inclusive para indivíduos que precisam organizar longas rotinas de atividades diárias e apresentam dificuldades na aprendizagem relacional.

\section{REFERÊNCIAS}

Assis, G. J. A. \& Sampaio, M. E. C. (2003). Efeitos de dois procedimentos de ensino na formação de classes seqüenciais. Interação em Psicologia, 7(2), 53-62

Catania, A. C. (1999). Aprendizagem: comportamento, linguagem e cognição. Porto Alegre: Artes Médicas Sul.

Green, G.; Stromer, R. \& Mackay, H. (1993). Relational learning in stimulus sequences. The Psychological Record, 43, 599-616. 
Holcomb, W. L.; Stromer, R. \& Mackay, H. A. (1997). Transitivity and emergent sequence performance in young children. Journal of Experimental Child Psychology, 65, 96-124.

Lazar, R. \& Kotlarchyk, B. J. (1986). Second order control of sequence class equivalence in children. Behavioral Proces, 13, 205-215.

Leite, S. A. S. (1984). Instrumento de avaliação do repertório básico para a alfabetização (IAR). São Paulo: Edicon.

Pilgrim, C. \& Galizio, M. (2000). Stimulus equivalence and units of analysis. Em J. C. Leslie \& D. Blackman (Orgs.), Experimental and Applied Analysis of Human Behavior (pp. 111-126). Reno, NV: Context Press.

Sampaio, M. E. C. \& Assis, G. J. A. (no prelo). Equivalência de estímulos após a formação de classes seqüenciais com portadores de necessidades educacionais especiais. Acta Comportamentalia.

Sidman. M. (1986). Functional Analysis of Emergent Verbal Classes. Em T. Thompson \& M. D. L. Zeiler (Orgs.), Analysis and Integration of Behavioral Units (pp. 213-245). N.J.: Lawrence Erlbaum Associates Publishers.

Sidman, M. (1992). Equivalence relations: some basic considerations. Em S. C. Hayes \& L. J. Hayes (Orgs.), Understanding verbal relations: the second and third international institute on verbal relations (pp. 15-27). Reno, NV: Context Press.

Sidman. M. (1994). Equivalence Relations and Behavior: a research story. Boston: Authors Cooperative, Inc. Publishers.

Sidman, M \& Tailby, W. (1982). Conditional discrimination versus matching to sample: An expansion of the testing para- digm. Journal of the Experimental Analysis of Behavior, 37, 522.

Sigurdardottir, Z. G.; Green, G. \& Saunders, R. R. (1990). Equivalence classes generated by sequence training. Journal of the Experimental Analysis of Behavior, 53, 47-63.

Skinner, B. F. (1992). Verbal Behavior. Acton, MA: Copley Publishing Group. (originalmente publicado em 1957).

Stevens, S. S. (1951). Mathematics, measurement, and psychophsics. Em S. S. Stevens (Org.), Handbook of Experimental Psychology (pp. 1-49). New York: John Wiley and Sons, Inc.

Stromer, R. \& Mackay, H. A. (1992a). Some effects of presenting novel stimuli on a child's sequence production. Experimental Analysis of Human Behavior Bulletin, 10, 21-25

Stromer, R. \& Mackay, H. A. (1992b). Conditional stimulus control of children's sequence production. Psychological Reports, 70, 903-912.

Stromer, R. \& Mackay, H. A. (1993). Human sequential behavior: relations among stimuli, class formation and derived sequences. The Psychological Record, 43, 107-131.

Enviado: 04/12/2003

Revisado: $12 / 08 / 2004$

Aceito: 15/09/2004

\section{Notas:}

${ }^{1}$ Os autores agradecem as sugestões e críticas ao trabalho pelo prof. Dr. Olavo de Faria Galvão e ao Sr. João Vicente do Nascimento pelo desenvolvimento do programa de análise de dados comportamentais.

${ }^{2}$ Sequence-production procedures often involve displays of two or more physically dissimilar visual stimuli and contingencies of reinforcement that require a specified sequence of responses to these stimuli, regardless of their spatial locations (Stromer \& Mackay, p. 21, 1992b).

\section{Sobre os autores:}

Grauben José Alves de Assis: Doutor em Psicologia Experimental. Docente no Departamento de Psicologia Experimental, Universidade Federal do Pará. Pesquisador II do CNPq. Endereço Eletrônico: gjaa@cpgp.ufpa.br.

Lívia Cristinne Arrelias Costa: Bolsista de Iniciação Científica (PIBIC-CNPq). 